\title{
Immunohistochemical study for relationship between vessel and lymphatic properties and tooth marks in human oral mucosa
}

\author{
Toru Inomata, ${ }^{1}$ Yoko Miwa, ${ }^{2}$ Shinichi Kawata, ${ }^{1}$ Takuya Omotehara, ${ }^{1}$ Iwao Sato, ${ }^{1}$ Masahiro Itoh ${ }^{1}$ \\ ${ }^{1}$ Department of Anatomy, Tokyo Medical University, Tokyo \\ ${ }^{2}$ Department of Anatomy, School of Life Dentistry at Tokyo Nippon Dental University, Tokyo, Japan
}

\begin{abstract}
Angiogenesis is an important issue related to normal growth and differentiation, and it is a critical issue in the progression of human disease in oral mucosa. Tooth marks occur after clenching the teeth for a long period under muscle tension in the human oral cavity. However, the sites of angiogenesis, cell differentiation and microvessel density are not known for human mucosa with tooth marks. Therefore, we investigated the relationship between the markers of proliferation (Ki-67), angiogenesis (CD31, D2-40, VEGF-A), and marks from teeth in the second molar region using immunohistochemical methods. In addition, we compared these areas with the mucous membrane. Our results revealed blood and lymphoid vessels in irregular mucosa structures, and the vessels in the oral mucosa were observed in three types of samples: dentulous, denture attachment (containing partial teeth), and edentulous samples. The localization of the angiogenesis was related to the structure of the oral mucosa of connective tissue in humans, such as the mucosal fold-like of the buccal region. Using principal component analysis (PCA), we found that tooth occlusal condition, gender, anti-VEGF-A reaction levels in oral mucosa of the epithelium were positive factors in all groups, which is in contrast to the negative association of Ki-67 reaction in the epithelium and CD31 expression. In addition, Ki-67 reaction in oral mucosa had negative impacts, in contrast to the positive association of D2-40. These PCA properties provide useful information for future study of tumour progression or mechanical stress in remodelling of oral mucosa and angiogenesis. Moreover, mechanical stress of the occlusal condition may be correlated with tumour angiogenic activity and cell differentiation in human oral mucosa.
\end{abstract}

Key words: Tooth mark; mucosa; CD31;D2-40, VEGF-A.

Correspondence: Iwao Sato, Department of Anatomy, Tokyo Medical University, 6-1-1, Shinjuku, Shinjuku-ku, Tokyo 160-8402, Japan, Tel. +81.3.33516141(231) - Fax:+81.3.33411137. E-mail: iwaoa1@tokyo-med.ac.jp

Contributions: All the authors made a substantive intellectual contribution, performed part of the experiments. All the authors have read and approved the final version of the manuscript and agreed to be accountable for all aspects of the work.

Conflict of interest: The authors declare that they have no competing interests, and all authors confirm accuracy.

Ethics approval and informed consent: The study was approved by Ethics Review Committee of Nippon Dental University (2018/21/ July; no. NDU-T2015-20) and Tokyo Medical University, Institutional Review Board (2019/01/ March; TMU, no. T2018-0060).

This study was performed in line with the principles of the Declaration of Helsinki (as revised in 2013). The cadavers used in the present study were donated to Tokyo Medical University and Nippon Dental University, Tokyo, Japan, based on the Act on Body Donation for Medical and Dental Education. All the donors willingly signed a form consenting to the body donation for education and research, and all the donors could revoke the intended donation any time without any disadvantages. The collection and use of mucosa data of all cadavers have been permitted by theirs and their family, and then carried out according to the Law Concerning Cadaver Dissection and Preservation enacted in Japan in 1949.

Availability of data and materials: The data used to support the findings of this study are available from the corresponding author on reasonable request. 


\section{Introduction}

Angiogenesis is an essential process in normal growth and differentiation. However, defective and excessive forms of angiogenesis are crucial events in the progression of human diseases. Angiogenesis has been largely investigated in psoriasis, arthritis, diabetic retinopathy and malignant tumours. ${ }^{1}$ The proliferation of endothelial cells, such as tip cells and stalk cells, is a key event during sprouting angiogenesis. ${ }^{2}$ Endothelial angiogenesis is also related to the interplay of cellular characteristics. ${ }^{3}$ Vascular endothelial growth factor (VEGF) expression in oral tissues is also associated with angiogenesis, disease progression and field cancerization. ${ }^{4}$ Moreover, tumour angiogenic activity also relates to the density of the vasculature. The quantitative or qualitative changes in the vascularity of tissue play important roles in the structure of oral mucosa. ${ }^{5}$ There is a close association between vascularity and tumour progression in the oral mucosa. ${ }^{5}$ The notable biomarker $\mathrm{Ki}-67$ is expressed at significantly different levels that are important for angiogenesis, which is an essential process involved in normal growth and differentiation. In contrast, tumour angiogenic activity also relates to the density of the vasculature. The notable biomarker Ki-67 exhibits significantly different expression levels that are important for the cancer transformation rate. ${ }^{6}$ There is a significant positive correlation between microvessel density and mast cell concentration in normal mucosa. Mast cells play a more important role in the regulation of angiogenesis in oral squamous cell carcinoma than in normal mucosa. ${ }^{7}$ These results indicated that angiogenesis, cell differentiation markers, and vessel density need to be investigated in oral mucosa in the progression of human diseases involving mechanical stress in dentures or in dentulous and edentulous subjects. Tooth marks are formed by strongly pressing the tongue or lip/buccal mucosa against the dentition. The lips and buccal mucosa generally form light contact with the teeth, and general dentitions do not form tooth marks. However abnormal dentition leads to tooth marks, which may affect the oral mucosa in angiogenesis and development of oral squamous cell carcinoma. Despite limited previous research, it is thought that tooth marks occur after a long period of clinching teeth due to muscle tension. The site of the oral mucosa is different in various palatine regions and the gingiva (squares, $400 \times 400 \mathrm{~mm}^{2}$ ) between the maxilla and mandible. ${ }^{8,9}$ These different effects in the distribution of sensory receptors in the oral mucosa affect functional vascular blood vessels. It is generally accepted that resulted from the normal occlusion or malocclusion of teeth, a tooth mark may lead to tumorigenicity changes in cell kinetics due to mechanical stress. Therefore, the tooth mark may be an important information for exploring the causes of the oral cancer, cell dynamics, or disease progression. Our investigation concerns the tooth marks effect to the cell dynamics, disease progression or cancerization. However, the appearance structure and distribution of CD31, D2-40, VEGF$\mathrm{A}$ and $\mathrm{Ki}-67$ in the human mucosa between tooth marks are not known in cell dynamics for the causes of the oral cancer. The mechanisms behind angiogenesis, cell differentiation and vessel density are not known in human mucosa. Therefore, we investigated the relationship between epithelial and oral mucosal characteristics. By using immunohistochemical method, the evaluations were carried out concerning the expressions of angiogenesis markers CD31, D2-40, and VEGF-A and a proliferation marker Ki-67 in human mucosa with tooth marks. Furthermore, the upper results were compared to those from normal mucosa. These data may provide basic information about tumour progression in the oral mucosa under mechanical stress from teeth and dentures.

\section{Materials and Methods}

\section{Sample preparation}

The present study examined the buccal mucosa of the lip in the first molar region of 18 human cadavers ranging from 75 to 89 years old (mean $78.1 \pm 12.1$ years old; male, $n=9,80.1 \pm 9.1$ years old; female, $n=9,84.6 \pm 15.1$ years old). We classified them into the following three groups: the dentulous group who maintained occlusal function (DG), the denture attachment with tooth mark group who contained partial teeth (DAT), and the edentulous group (EG) (each, male $n=3$, female, $n=3$ ). All human cadavers used in the study were donated for dissection. The samples were injected with $10 \%$ formalin with return perfusion via the femoral artery. For macroscopic observation, human oral mucosae of the lip were selected from the buccal mucosa of molar region in three types of DG, DAT and EG samples. We investigated the morphological structure of mucosa membranes in three types. The samples collected from the oral mucosa at the first molar region of the human buccal mucosa of the lip were used for immunohistochemical analyses. In the microscopic observation, the mucosal lamina propria on the surface of the epithelium and the inner side of the mucosal lamina propria was classified into three types; developed lamina propria (DLP) with histologically fold-like mucosa, irregular lamina propria (ILP) with small fold-like mucosa, and flat lamina propria (FLP) with smooth mucosa in the human buccal mucosa of the lip. We also measured ration of these three types in DG, DAT and EG sections (each of the 60 measurement points).

\section{Paraffin-embedded sections of human oral mucosa}

The lips of human cadavers were fixed with tissue fixative (Genostaff Co., Ltd., Tokyo, Japan), embedded in paraffin and cut into 5 - $\mu \mathrm{m}$ sections. The sections, which were vertical from the tooth axis to the lip, were deparaffinized with xylene and dehydrated with a series of ethanol solutions in phosphate-buffered saline. Endogenous peroxidase was blocked with $0.3 \% \mathrm{H}_{2} \mathrm{O}_{2}$ in methanol for $30 \mathrm{~min}$, and then the sections were blocked with Protein Block (G-Block, Genostaff Co., Ltd. Tokyo) and an avidin/biotin blocking kit (Vector SP-2001). Samples were incubated overnight at $4{ }^{\circ} \mathrm{C}$ with primary rabbit polyclonal antibodies against CD31 (1:1,000; CD31 mouse mAb Dako M0823, Glostrup, Denmark), D2-40 (1:1,000; Anti D2-40 mouse mAb Nichirei 413451, Tokyo, Japan), anti-VEGF-A (1:1,000; Ultra Clone Ltd., UK), anti-Ki-67 (1:1,000; Ki-67 mouse mAb Dako M7240) or normal goat serum as the negative control (left fascia samples). The sections were washed with $0.05 \mathrm{M}$ Tris-buffered saline (TBS), pH 7.5, TBS with addition of $0.05 \%$ of non-ionic surfactant Tween-20 (TBST), $\mathrm{pH} 7.5$, and TBST, $\mathrm{pH}$ 9.0. Then they were incubated with a biotin-conjugated goat anti-rabbit IgG (Dako E0432) at room temperature for $30 \mathrm{~min}$. Sections were washed with TBST and TBS and incubated with peroxidase-conjugated streptavidin (Nichirei) at room temperature for $5 \mathrm{~min}$. Peroxidase activity was visualized using diaminobenzidine. The sections were counterstained with Mayer's haematoxylin (Muto Pure Chemicals Co., Ltd., Tokyo, Japan), dehydrated, and mounted with malinol (Muto Pure Chemicals Co., Ltd.). Images were acquired using a microscope (Leica DM 2500 16; Leica Microsystems, Buffalo Grove, IL, USA) and Leica Application Suite software (Leica Microsystems, Wetzlar, Germany). We also measured the number of positive cells and the reaction area in each section using random sampling methods for each small area $\left(0.25 \mathrm{~mm}^{2}, \mathrm{n}=20\right)$ and an image analyser. 


\section{Statistical analysis}

In the measurement data for FLP, DLP and ILP, we randomly selected in each of the 60 squares $\left(400 \times 400 \mathrm{~mm}^{2}\right)$ from DG, DAT and EG. The measurement data of immunopositive cells in the oral mucosa were assessed using Pearson's chi-square test to test the validity of a distribution assumed for occlusion loss. The results are expressed as percentages. The level of significance $(\mathrm{P})$ was calculated using Person's chi-square test $(\mathrm{P}<0.001)$. The statistical analyses were performed using IBM SPSS Statistics Base, ver. 22 (IBM, Chicago, IL, USA). The differences in the quantitative immunohistochemical data among the groups were assessed using one-way analysis of variance (ANOVA) followed by Bonferroni's post-hoc test. The level of significance was set as $\mathrm{P}<0.05$. The results are reported as the mean $\pm \mathrm{SD}$. Furthermore, we performed multivariate modelling of our quantitative data for its use in the principal component analysis (PCA), which was used to estimate the interaction between the effects of morphological tooth marks and dentures within the groups; these effects were assessed using one-way analysis of variance (ANOVA) followed by Bonferroni's post-hoc test with one categorical independent variable and one continuous variable (the independent variable can consist of a number of groups). The PCA was performed for human mucosa considering 11 elements: immunohistochemical elements (Ki-67, CD31, D2-40, and VEGF-A), age, gender, mucosa structure concerning the surface of epithelium (SE), mucosal lamina propria (MLP), and teeth (DG, DAT, EG). Then, we performed a cluster analysis (CA) using average linkage between groups (hierarchical cluster analysis algorithms) based on the significant components of the PCA analysis that was performed on individuals for two divided clusters. ${ }^{10}$

\section{Analysis of images after immunolabeling for CD31, D2-40, VEGF-A and Ki-67 reactions}

We acquired all digital images from each 6 slide of DG, DAT and EG that were scanned at an absolute magnification of $400 \times$ [resolution of $0.25 \mathrm{~mm} / \mathrm{pixel}(100,000 \mathrm{pix} / \mathrm{in}$.)] (each 30 squares); the positive control was a human lip, and a pixel density analysis method was used. ${ }^{11}$ The background illumination levels were also calibrated using a prescan procedure. Each stain was individually calibrated via analysis of single-stained sections and the recording of optical density vectors, such as staining colour. A localization algorithm used the deconvolution method to separate the stains and measured each pixel according to the number of stains present. The algorithm also provided a greencolour mark-up image for visualization of a positive reaction (Figure $1 \mathrm{a}, \mathrm{b}$ ).

\section{Results}

\section{Macroscopic observations}

The human oral mucosa structure of the lip exhibited specific characteristics in the anterior and posterior regions (buccal mucosa of molar region) of the DG, DAT and EG samples. A developed mucous membrane with fold-like structural mucosa was found in the DG (Figure 1c), and a flat and thin oral mucosa with small fold-like structural mucosa was found in the DAT samples (Figure 1d). Irregularly arranged mucosa membranes with and flat mucosa surface structures were also found in the EG samples (Figure 1e). Specific tooth marks on the oral mucosa were primarily obtained, such as crown tooth impressions in the DG or DAT samples. The crown tooth impressions were clearly found in the oral mucosa from the anterior to posterior regions in the DG samples, and these tooth marks were stronger in the incisor region than in the molar region of the mucosa at macroscopic levels (Figure 1c). However, a developed mucosal foldlike structure was primarily found in the posterior region compared to the incisor region of the oral mucosa of the maxilla and mandible of the DG (Figure 1c). A developed mucosal fold-like structure was rarely found upon macroscopic observation of the EG samples, and the human oral mucosa structure of the lip exhibited specific characteristics at the anterior and posterior regions (buccal mucosa of molar region) in the DG, DAT and EG samples. According to the morphological structure of the mucosal lamina propria on the surface of the epithelium and the inner side of the mucosal lamina propria, the lamina propria were divided into three types: DLP, FLP, and FLP. The ratio for each of the three types was different in the DG, DAT and EG samples.

In the EG sections, the FLP type is mainly found on the surface of the epithelium $(70.0 \%, 42 / 60)$ and the DLP $(20.0 \%$, $12 / 60)$. In the DAT sections, the FLP type was mainly found on the surface of the epithelium $(61.7 \%, 27 / 60)$, the DLP $(21.7 \%$, $13 / 60)$, and the ILP $(16.7 \%, 10 / 60)$. In the EG sections, the FLP type was mainly found in the mucosal lamina propria $(21.7 \%$, $13 / 60)$, the DLP $(11.7 \%, 7 / 60)$, and the ILP $(71.7 \%, 43 / 60)$.

\section{Light microscopic observations}

Vertical sections were generated for histochemical observations of the human oral mucosa of lips at anterior and posterior regions (buccal mucosa of molar region) of the DG, DAT and EG (each $n=3$ ) samples, and they were compared by hematoxylineosin staining. A developed mucous was found at the mucosal fold-like structure of the buccal region in the DG sections (Figure $1 \mathrm{f}, \mathrm{g}$ ). A developed papilla of epithelial mucosa was also found in the buccal mucosa of the buccal region in the DG sections. In contrast, a somewhat thick smooth surface of buccal mucosa with irregular papilla of the epithelial mucosa was primarily found in the DAT sections (Figure 1 h,i). An irregular surface of the buccal mucosa with no fold-like formation was found in the EG sections (Figure $1 \mathrm{j}, \mathrm{k}$ ). However, numerous flat cells were scattered in the mucosal lamina propria of the DAT sections (Figure $1 \mathrm{~h}, \mathrm{i}$ ).

\section{Distribution of CD31-positive vessel}

Large and small vessels that were CD31-positive reaction (blood vessels) had developed and were concentrated at the submucosa and lamina propria of the buccal region in the DG and EG sections (Figure 2 a,b,q,r). In particular, numerous CD31-positive vessels were concentrated in the mucosal lamina propria beneath the basal layer of a developed mucosal fold-like structure in the epithelium of the buccal region in the DG sections (Figure $2 \mathrm{a}, \mathrm{b}$ ). The CD31-positive vessels were located beneath or in the papilla of the epithelial mucosa of the buccal region in the EG sections (Figure 2 q,r). In contrast, some small vessels were scattered in the mucosal lamina propria beneath the basal layer of the epithelium of the buccal region in the DAT sections (Figure $2 \mathrm{i}, \mathrm{j}$ ). Large vessels were primarily located at the lamina propria near the muscle layer of the buccal region in the DAT sections (Figure 2r).

\section{Distributions of D2-40-positive vessels}

D2-40-positive vessels (lymphoid vessels) were primarily found in the submucosa and reticular layers (Figure 2). Many small and large expanded or thin elongated D2-40 positive vessels were found at the submucosa and lamina propria of the buccal region in the DG sections, and these vessels were concentrated at the mucosal lamina propria beneath the basal layer of a developed mucosal fold-like structure of epithelium in the DG sections (Figure $2 \mathrm{c}, \mathrm{d}$ ). Large D2-40-positive vessels were observed as elongated or expanded structures at the submucosa and lamina propria of the 


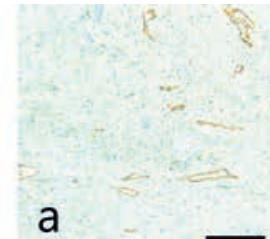

a
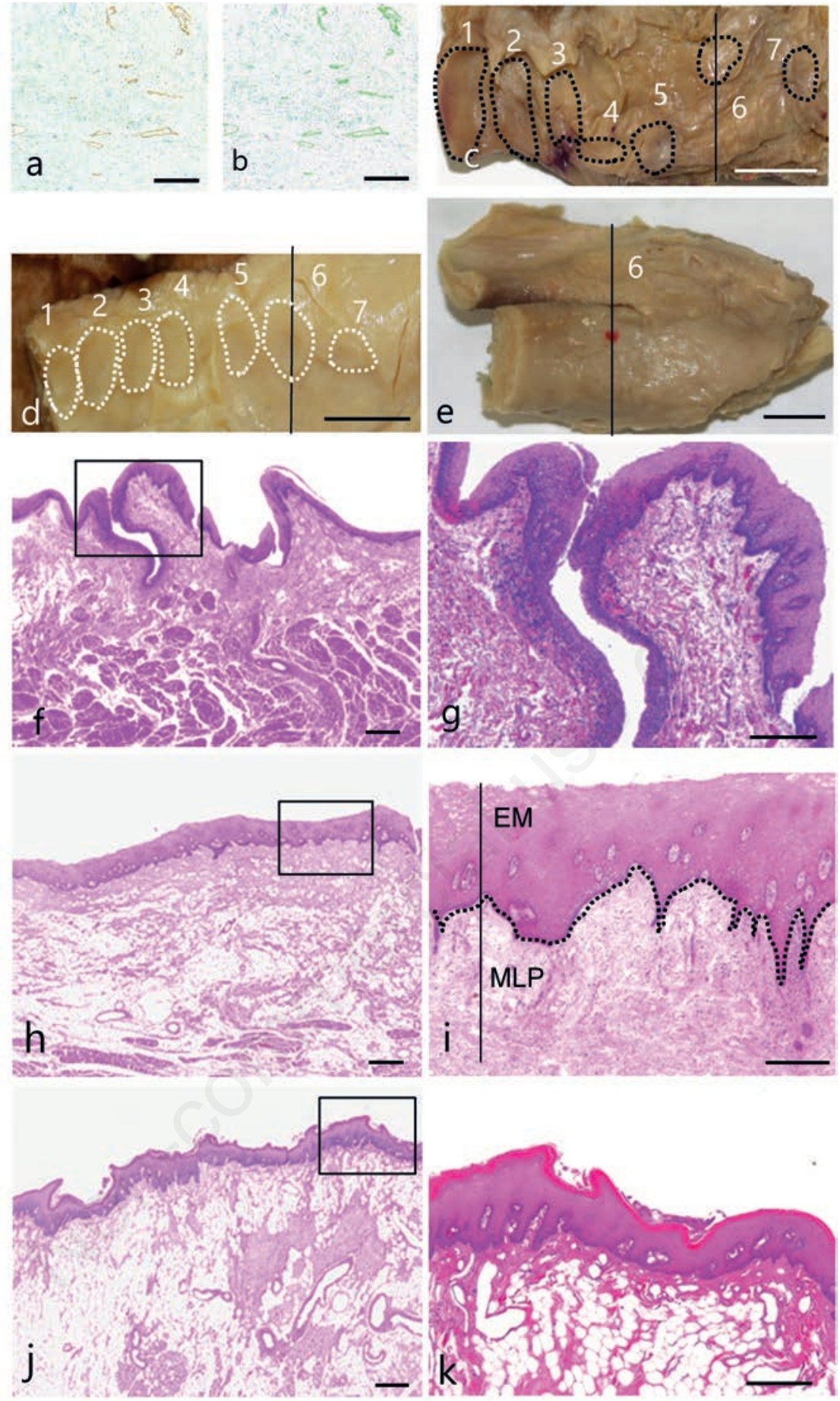
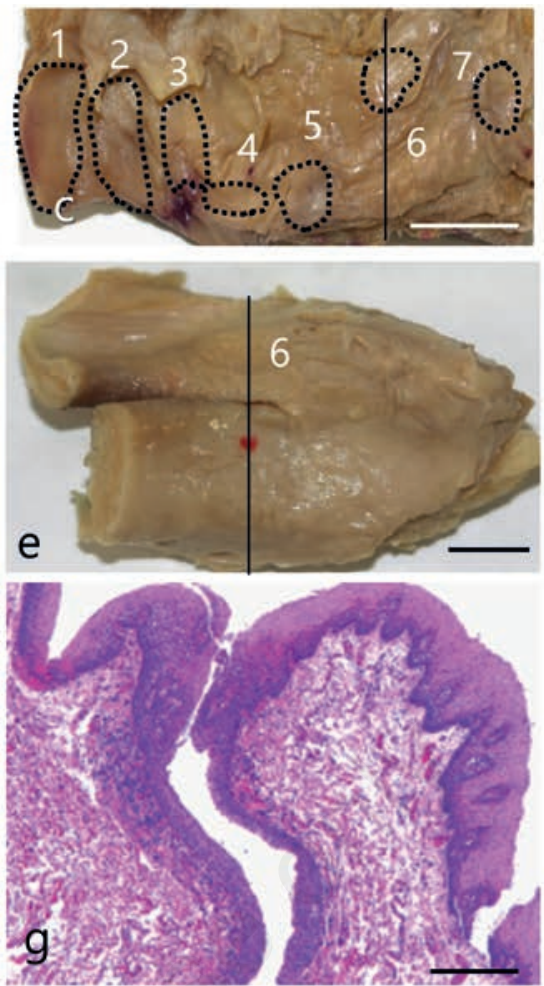

Figure 1. Oral mucosa. a,b) Digital images of anti-D2-40-stained sections (a) were scanned using an algorithm for pixel density analysis modified by Krajewska et al.:11 the algorithm provided a green-colour-marked image of the immunopositive parts (b). Tooth marks on the inner surface of the oral mucosa from the upper jaw (maxilla) are present in the dentulous group (DG) (black dot lines in c); they are also shown in correspondence to the denture attachment or along the border of denture impressions in the lower jaw containing partial teeth (DAT) (white dot lines in d); obviously, no tooth mark could be observed in the upper and lower jaws of the edentulous group (EG) (e). Numbers 1 to 7 in panels (c), (d), and (e) indicate each tooth position. The red spot in panel e) indicates the measurement position at the first molar equivalent point, while the vertical line represents the landmark of the frontal sections shown in panels (f) to (k). A developed mucous was found in the oral mucosa of DG (c,f,g), a flat and thin oral mucosa with fat cells was observed in DAT, and an irregularly arranged mucosal membrane was detected in EG. The morphological structure of the mucosal lamina propria on the surface of the epithelium and the inner side of the mucosal lamina propria was classified into three types: developed lamina propria $(f, g)$, irregular lamina propria $(j, k)$, and plate lamina propria $(h, i)$. $f, h, i)$ Low magnification of mucosal tissue sections from the upper lips of DG (f), DAT (d,h,i), and EG (e,i,j,k). g,i,k) High magnification of the square areas labelled in panels $f, h$ and $j$, respectively. In panel $i$, the dot line indicates the border between the epithelial mucosa layer (EM) and mucosal lamina propria layer (MLP). Arrows in panel f) indicate the mucosal fold-like structure. Scale bars: a,b) $100 \mu \mathrm{m}$; c-e) $1 \mathrm{~cm}$; f,h,j) $300 \mu \mathrm{m}$; g,i,k) $200 \mu \mathrm{m}$. 
buccal region in the DAT sections (Figure $2 \mathrm{k}, 1$ ). However, D240-positive vessels were very rare in the submucosa and lamina propria of the buccal region of the EG sections (Figure $2 \mathrm{~s}, \mathrm{t}$ ).

\section{Distribution of VEGF-A positive cells}

VEGF-A-positive cells were primarily found in the submucosa (Figure 2). A few small VEGF-A-positive cells were found in the submucosa and lamina propria of the buccal region in the DG sections, and these positive cells were scattered in the mucosal lamina propria of a developed mucosal fold-like structure in the epithelium of the buccal region in the DG sections (Figure 2 e,f). VEGF-A-positive cells were rare in the submucosa and lamina propria of the buccal region of the DAT sections (Figure $2 \mathrm{~m}, \mathrm{n}$ ). However, VEGF-A-positive cells and vessels were found in the submucosa and lamina propria of the buccal region in the EG sections (Figure $2 \mathrm{u}, \mathrm{v}$ ).

\section{Distribution of anti-Ki-67 positive cells}

Ki-67-positive cells were primarily found in two regions (squamous epithelium and submucosa) of the human lip (Figure 2). Some Ki-67-positive cells were primarily found in the squamous epithelium of DG sections (Figure 2 g,h). Ki-67-positive cells were rare in the submucosa of the buccal region in the DAT sections (Figure 2 o,p). Numerous Ki-67-positive cells were primarily found in the squamous epithelium of the buccal region in the EG sections, while there were few Ki-67-positive cells in the submucosa (Figure $2 \mathrm{w}, \mathrm{x}$ ).

\section{Properties of immunohistochemical analysis data and PCA map between DG, DAT and EG}

The ratio of CD31 positive cells per field of the DG and EG are significantly higher than of the DAT $(\mathrm{P}<0.01)$ (Figure $3 \mathrm{~A})$. The ratio of D2-40-positive DG cells was also higher than what was observed in the DAT and EG $(\mathrm{P}<0.01)$. In contrast, the ratio of VEGF-A-positive cells in the connective tissue area of the DAT was significantly higher $(\mathrm{P}<0.01)$ than it was in the other areas (Figure 3A). PCA was performed for human mucosa in different groups for the following eleventh variables: Ki-67, CD31, D2-40, VEGF-A, ageing, gender, mucosa structure and dentures. The CD31, Denture and D2-40 elements were same positive relation groups, in contrast to that of negative relation groups such as the $\mathrm{Ki}-67 \mathrm{C}$ and aging. In contrast, mucosa of the epithelium
(ME), VEGF and gender were also same correlative groups in contrast to negative groups of the $\mathrm{Ki}-67 \mathrm{E}$ and mucosa connective tissue (MCo) in the PCA map (Figure 3A).

\section{Correlation coefficients between measured elements from human mucosa}

The following correlations among age data are shown in Table 1. We observed a negative correlation between Ki-67-positive reaction cells of the epithelium (Ki-67E) and anti-VEGF-A reaction in connective tissue (VEGF-C) areas $(\mathrm{P}<0.01 ;-0.530)$ and D2-40 $(\mathrm{P}<0.05 ;-0.355)$. A negative correlation was also observed between the Ki-67-positive cell ratio in the connective tissue $(\mathrm{Ki}-67 \mathrm{C})$ and VEGF-A in the epithelium (VEGF-E) $(\mathrm{P}<0.05 ;-0.367)$ and the VEGF-C $(\mathrm{P}<0.01 ;-0.413)$ areas. The tooth factors such as DG, DAT and EG (denture) were positively correlated with CD31 $(\mathrm{P}<0.01 ; 0.742)$ and $\mathrm{D} 2-40 \quad(\mathrm{P}<0.05$; $0.344)$, in contrast to the negative correlation with $\mathrm{Ki}-67 \mathrm{C}$ $(\mathrm{P}<0.05 ;-0.357)$ data. The mucosa of the ME was positively correlated with VEGF-E $(\mathrm{P}<0.05 ; 0.291)$ and denture $(\mathrm{P}<0.01$; $0.136)$, in contrast to the negative correlation with Ki-67C $(\mathrm{P}<0.05 ;-0.331)$. The MCo data were positively correlated with the denture data $(\mathrm{P}<0.05 ; 0.167)$. The ageing elements were positively correlated with $\mathrm{D} 2-40(\mathrm{P}<0.01 ; 0.468)$ and $\mathrm{Ki}-67 \mathrm{E}$ $(\mathrm{P}<0.05 ; 0.390)$ and $\mathrm{Ki}-67 \mathrm{C}(\mathrm{P}<0.01 ; 0.538)$; in contrast, ageing had a negative correlation with $\mathrm{CD} 31(\mathrm{P}<0.01 ;-0.488)$, the denture $(\mathrm{P}<0.01 ;-0.695), \mathrm{ME}(\mathrm{P}<0.01 ;-0.243)$ and $\mathrm{MCo}(\mathrm{P}<0.01$; $0.225)$. The gender elements were positively correlated with the denture $(\mathrm{P}<0.01 ; 0.105)$, the ageing elements $(\mathrm{P}<0.01 ; 0.126)$, VEGF-E $(\mathrm{P}<0.05 ; 0.273)$ and $\mathrm{ME}(\mathrm{P}<0.01 ; 0.281)$ in contrast, gender had a negative correlation with Ki-67-E $\mathrm{P}<0.01$; -0.761$)$, Ki-67C $(\mathrm{P}<0.01 ;-0.355)$, and the MCo $(\mathrm{P}<0.01 ;-0.379)$.

\section{PCA for human mucosa in different groups for Ki-67, CD31, D2-40, VEGF, age, gender, mucosa structure and dentures}

The variables were plotted in a two-dimensional space defined by two axes: component 1 (x-axis) and component 2 (yaxis). The two principal components significantly explained $57.6 \%$ (component $1,40.7 \%$; component $2,16.9 \%$ ) of the information in the data set of the human mucosa.

Figure $3 \mathrm{~B}$ shows a display of the human mucosa variables

Table 1. Correlation coefficients between measurement elements from oral mucosa.

\begin{tabular}{|c|c|c|c|c|c|c|c|c|c|c|c|}
\hline & VEGF-E & VEGF-C & CD31 & D2-40 & Ki-67-E & Ki-67-C & Denture & ME & MCo & Ageing & Gender \\
\hline VEGF-E & 1.00 & & & & & & & & & & \\
\hline VEGF-C & -0.002 & 1.00 & & & & & & & & & \\
\hline CD31 & 0.018 & -0.047 & 1.00 & & & & & & & & \\
\hline D2-40 & 0.56 & 0.079 & 0.250 & 1.00 & & & & & & & \\
\hline Ki-67-E & -0.279 & $-0.530 * *$ & 0.272 & $-0.355^{*}$ & 1.00 & & & & & & \\
\hline Ki-67-C & $-0.367^{*}$ & $-0.413^{* *}$ & -0.180 & $-0.390^{*}$ & 0.269 & 1.00 & & & & & \\
\hline Denture & 0.035 & 0.189 & $0.742 * *$ & $0.344^{*}$ & 0.101 & $-0.357^{*}$ & 1.00 & & & & \\
\hline $\mathrm{ME}$ & $0.291 *$ & 0.906 & 0.167 & 0.193 & -0.262 & $-0.331^{*}$ & $0.136^{* *}$ & 1.00 & & & \\
\hline $\mathrm{MCo}$ & -0.030 & -0.080 & -0.066 & 0.142 & 0.195 & 0.258 & $0.167^{*}$ & 0.009 & 1.00 & & \\
\hline Ageing & -0.197 & -0.158 & $-0.488^{* *}$ & $-0.359^{*}$ & $0.390^{*}$ & $0.538 * *$ & $-0.695^{* *}$ & $-0.243^{* *}$ & $-0.225^{* *}$ & 1.00 & \\
\hline Gender & $0.273^{*}$ & -0.018 & -0.275 & 0.093 & $-0.761^{* *}$ & $-0.367^{*}$ & $0.105^{* *}$ & $0.281^{* *}$ & $-0.379^{* *}$ & $0.126^{* *}$ & 1.00 \\
\hline
\end{tabular}

*Significant at $\mathrm{P}<0.05$; ** significant at $\mathrm{P}<0.01$ by Person's correlation coefficient; CD31, anti-CD31 positive reaction cell; Ki-67E, anti-Ki-67 positive reaction in oral epithelium area; Ki-67C, anti-Ki-67 positive reaction in in oral submucosa area; D2-40, anti-D2-40 reaction; VEGF-E, positive reaction of anti-VEGF-A in oral epithelium area, VEGF-C, anti-VEGF-A positive reaction in oral submucosa area; ME, epithelial mucosa structure (three grades such as developed, irregular, and flat); and MCo, mucosal lamina propria structure (three grades such as developed, irregular, and flat). 
and components 1 and 2 on a two-dimensional map (Table 2). We also observed positive contributions from VEGF-C (0.755), gender (0.627), ME (0.563), D2-40 (0.541) and the VEGF-E (0.505), and we observed negative contributions from cell differentiation
(Ki-67C, -0.755; Ki-67E, -0.614) in component 1. In contrast, component 2 was defined by the expression of CD31 (0.888), denture (0.804) and Ki-67E (0.556), while negative contributions were observed for gender (-0.701) (Table 2).
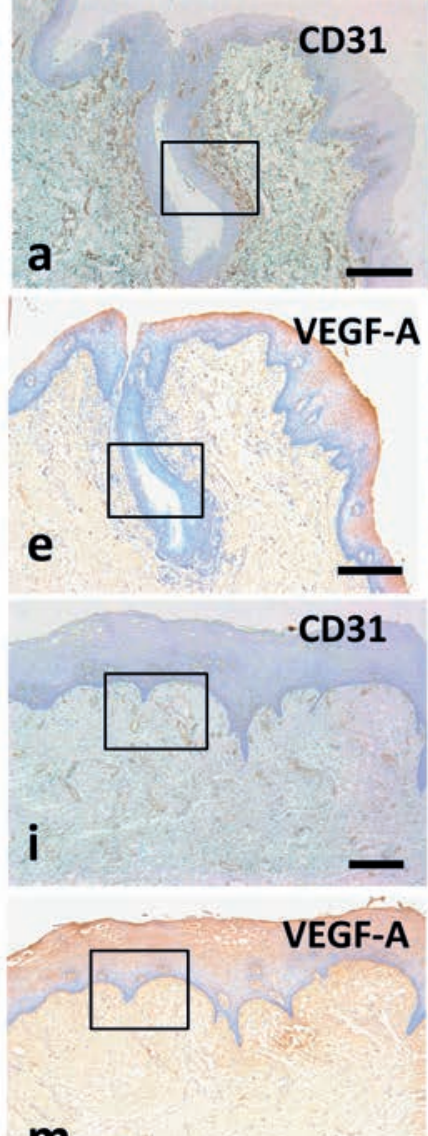

m

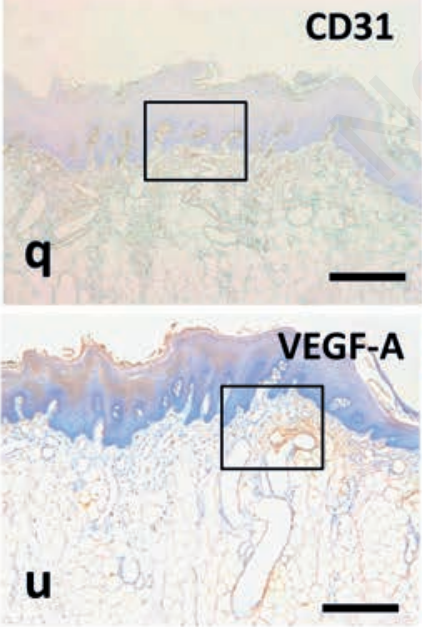

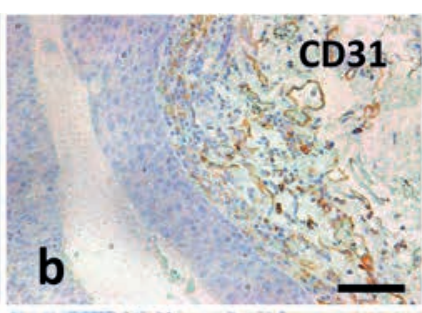
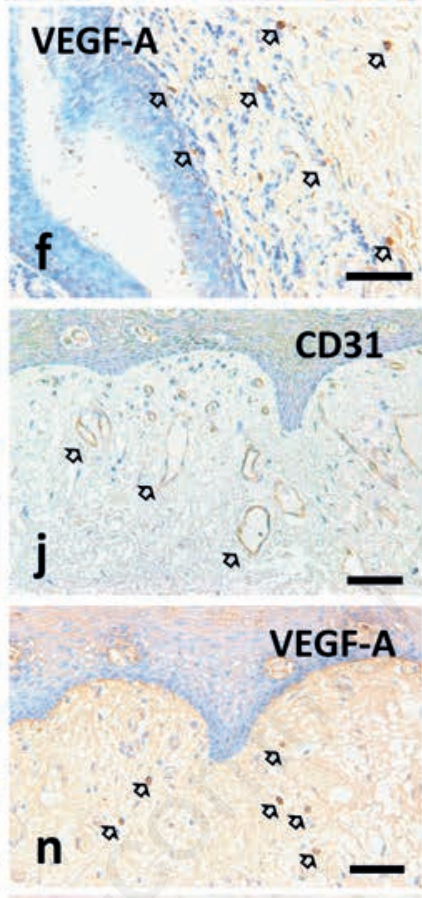
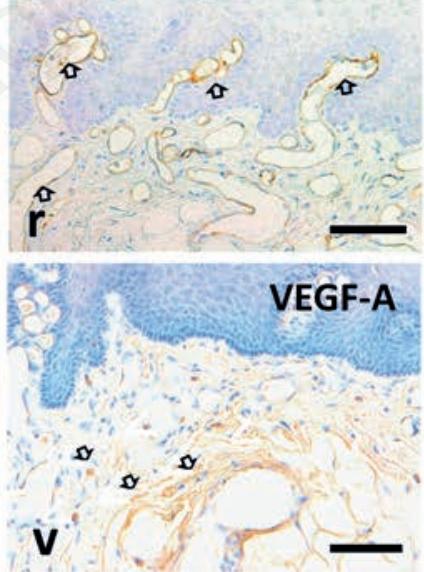
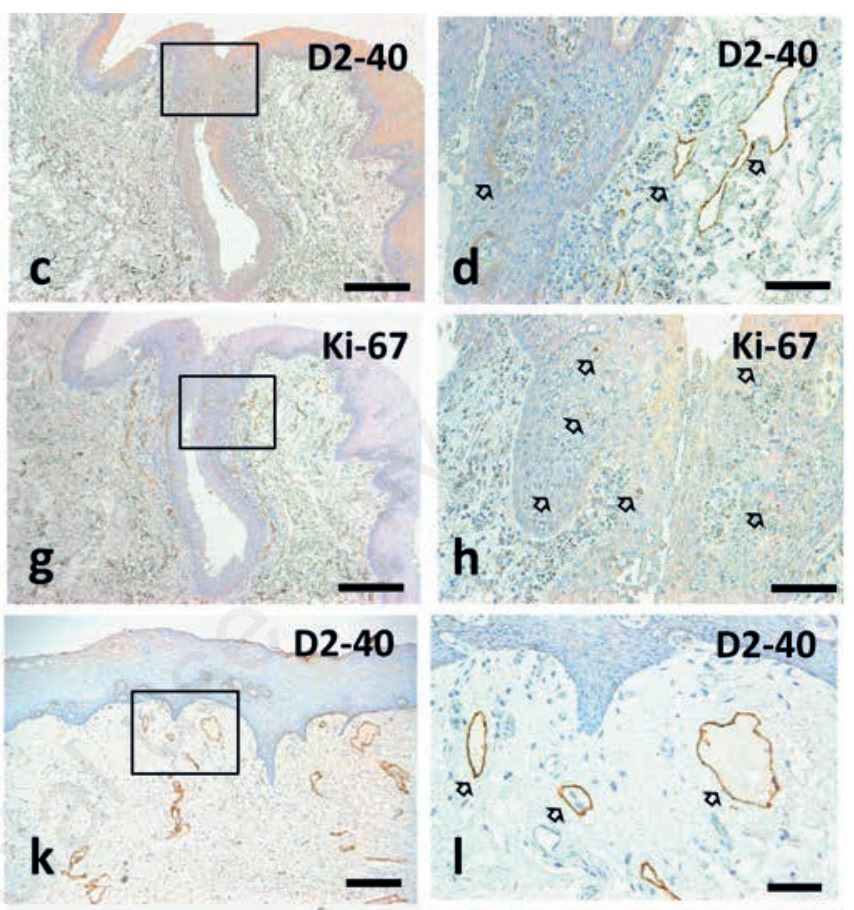

Ki-67

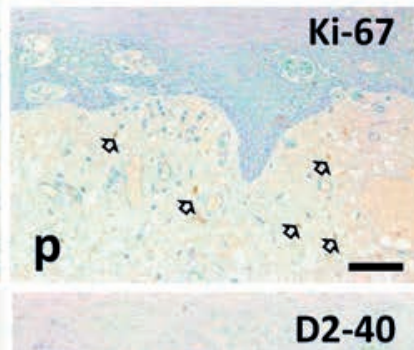

D2-40
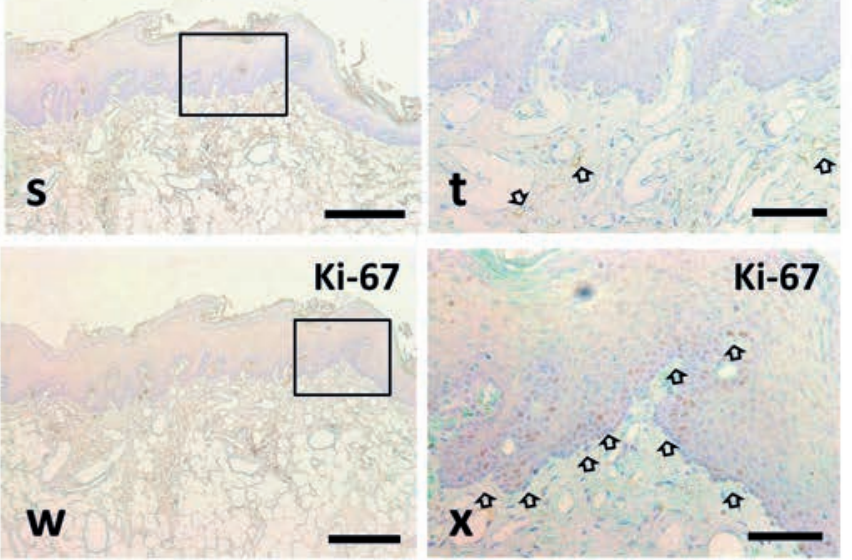

Figure 2. Anti-CD31, D2-40, VEGF-A, and Ki-67 immunoreactions in serial sections of the mucosa from the DG (dentulous) group (ah); the DAT (denture attachment with tooth mark) group (i-p); and the EG (edentulous) group (q-x). Positive signals for the different proteins are indicated by thick arrows. The panels in the second and fourth column are high magnifications of a square areas labelled in the corresponding left panels. Scale bars: panels in the first and third column, $200 \mu \mathrm{m}$; panels in the second and fourth column, $70 \mu \mathrm{m}$. 


\section{Discussion}

\section{Localization and density of blood vessels}

Morphometric analyses reflecting microvascular volume are more informative than the currently popular analysis of microvascular density. ${ }^{5}$ In general, more dense collections of microvessels are found in intralesional and perilesional regions of the oral squamous cell carcinoma (OSCC) compared to normal mucosa. There are significant positive correlations between microvessel density and mast cell concentration in OSCCs. ${ }^{7}$ Our results demonstrated that the density of CD31-positive vessels is an important element to the tooth marks and developed mucosal fold-like structure in the oral mucosa differentiation. The CD31-positive vessels increase significantly $(\mathrm{P}<0.001)$ with disease progression from normal oral mucosa to early and late carcinoma. ${ }^{1}$ The microvessel density is significantly increased in a relatively large spectrum of premalignant squamous cell lesions in the oral mucosa or skin. ${ }^{1}$ These results indicate that the developed mucosa histologically foldlike structure also reflect the vessel density in oral mucosa. The CD31positive vessel density may trigger mutations in cells on the surface of the buccal mucosa in the human lip. Moreover, our analysis revealed a significant difference between the dentulous and denture sample with tooth marks in the oral mucosa. The microvessel density reflects inflammation due to occlusal stress in the oral mucosa. Irregularly occlusal of the dentulous sample and nonconforming dentures edentulous sample may exhibit CD31-positive vessel density due to different structures in the oral mucosa. Therefore, CD31-positive vessel is an important element such as the tooth marks and developed fold-like oral mucosa. There is a significant positive correlation between vessel density and mast cell concentration in normal mucosa. ${ }^{7}$ In our results, numerous fat cells mainly observed in the HE stained sections from the DAT and EG samples. Pyziak et al. ${ }^{7}$ suggested that mast cells play a more important role in the regulation of angiogenesis in oral squamous cell carcinoma. Therefore, cell differenced marker and vessel density may be one of the most important markers in oral squamous cell carcinoma. In contrast, the density of lymphoid vessels had almost no difference among the three types. D2-40-positive vessels were found in the submucosa and lamina propria of the buccal region in the DG and DAT sections, and they formed a mucosal fold-like structure in the buccal region. The specific tooth marks on the oral mucosa, such as from dentures, affected cell differentiation, as indicated by Ki-67-positive reaction in the DAT sections. The expression of Ki67 was significantly different in oral submucous fibrosis, which is characterized by a juxtaepithelial inflammatory reaction and progressive fibrosis of the submucosal tissues, and normal oral mucosa samples. ${ }^{6}$ The expression of $\mathrm{Ki} 67$ is also identified in endothelial cells, which confirms the proliferative status of the microvascular bed in the mucosa of the mandibular edentulous ridge. ${ }^{12}$ The localization of Ki67-positive cells was primarily in submucosal tissues. The Ki-67-positive cells were primarily found in the epithelial layer and the submucosal tissues, and conversely, the presence of dentures following the loss of teeth (denture attachment sample) impacted on the oral submucosal tissue. The presence of Ki67-positive cells was related to the metabolic system in the epithelial layer of the oral mucosa in the EG. Therefore, there is a possibility that teeth loss and the cell differentiations may reflect a reduction in the occlusal. Tsuruoka et al. ${ }^{13}$ indicated that mechanical compression of the palatal plate induced ischaemia due to damage in blood flow, and VEGF-A-positive cell is synthesized to maintain homeostasis in the denture-supporting tissue, including the periosteum, according to rat experimental data that used these conditions. However, the mechanical stress from teeth is different from denture compression conditions because of the different conditions, such as a blood flow, in the examined bone and epithelial tissues of the rat palate. This study only shows that mechanical stress inhibits the blood supply system via compression. The DAT resembled the DG, which induced CD31-positive ves-
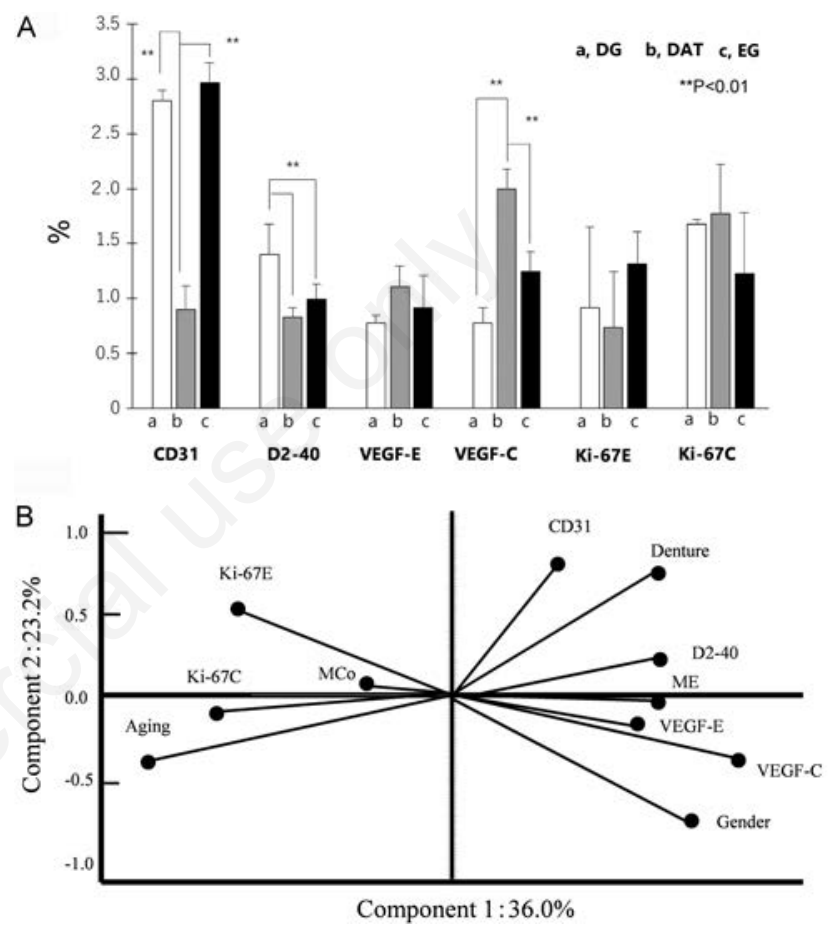

Figure 3. A) Percentage of cells positive for CD31, D2-40, VEGFA (at the surface of epithelium, VEGF-E; or in the mucosal lamina propria, VEGF-C), or Ki67 (at the surface of epithelium, Ki-67E; or in the mucosal lamina propria, Ki-67C), in the DG (a), DAT (b), and EG (c) from human lip mucosa; ${ }^{* *} \mathbf{P}<0.01$ by two-way ANOVA with Bonferroni's post-hoc test. B) Variable factor map plotted based on PCA of measured immunohistochemical levels data, aging, and gender. The original variables are the projected dimension space defined by component 1 (x-axis) and component 2 (y-axis). CD31, CD31-positive reaction; Ki-67E, anti-Ki-67-positive reaction in oral epithelium area; $\mathrm{Ki}-67 \mathrm{C}$, anti-Ki-67-positive reaction in mucosal lamina propria; $\mathrm{D} 2-40$, anti-D2-40- positive reaction; VEGF-E, positive reaction for VEGF-A in the oral epithelium area; VEGF-C, positive reaction for VEGF-A in mucosal lamina propria; ME, epithelial mucosa structure (three grades such as developed, irregular, and flat); and MCo, mucosal lamina propria structure (three grades such as developed, irregular, and flat).

Table 2. Components matrices between measurements elements from human mucosa in Components 1 and Components 2.

\begin{tabular}{lcccccccccrrr} 
& Aging & VEGF-C & Ki-67C & Ki-67E & ME & D2-40 & VEGF-E & CD31 & Denture Gender & MCo \\
Components 1 & -0.899 & 0.755 & -0.706 & -0.614 & 0.564 & 0.541 & 0.505 & 0.284 & 0.544 & 0.627 & -0.263 \\
Components 2 & -0.296 & -0.326 & -0.027 & 0.556 & -0.02 & 0.294 & -0.172 & 0.888 & 0.804 & -0.701 & 0.072 \\
\hline
\end{tabular}

CD31, anti-CD31 positive reaction cell; Ki-67E, anti-Ki-67-positive reaction cells of the epithelium; Ki-67C, Ki-67 positive reaction cell in the connective tissue; $\mathrm{ME}$, mucosa of the epithelium; MCo, mucosa connective tissue; denture, tooth factors such as in DG, DAT, and EG; VEGF-C, anti-VEGF-A positive reaction cell in connective tissue; and VEGF-E, anti-VEGF-A in the epithelium. 
sels and D2-40-positive vessels, such as lymphoid vessels. Notably, mechanical stress may cause cell mutations, such as those that cause cancer. We examined the distribution and density of VEGF positive reaction in the three groups of denture samples, and the VEGF-A expression density in the DAT was significantly greater than it was in the other groups. Carlile et al. ${ }^{4}$ also reported that VEGF expression in the oral mucosa was not associated with angiogenesis, field cancerization or transition to dysplasia. However, this marker is not useful in different dentures of the human oral mucosa. Our results suggest that CD31, D2-40 and VEGF-A related microvessel density makers differed in denture condition, and tooth marks and histologically foldmucosa are a related element in the DG to the tumour angiogenic activity and cell differentiation compared to other denture condition groups.

\section{PCA analysis and correlation of the immunohistochemical results}

The properties of the immunohistochemical analysis indicate various patterns for each angiogenic and cell differentiation marker in our results. We observed that the tooth marks mainly appeared in the DG and DAT. In the connective tissue beneath, the epithelial layer was lower, and cell activity was low except for the production of CD31 and VEGF-A. The effect of these factors on developing blood vessels and their quantity was related to the tissue repair and development of organs, despite the uncontrolled angiogenesis effect that these factors have on tumours and other diseases. Well-balanced angiogenesis is important for cell differentiation. In our results, the ED, DAT and DG have different morphological structures, such as tooth marks; however, these morphological properties mainly appeared during cell differentiation in the mucosal lamina propria, which is rich with blood vessels. Our correlation results also indicated that positive aging elements is reflected to $\mathrm{Ki}-67$-positive cells density in the epithelial layer in contrast to that of the negative gender elements to the Ki-67 positive cells density in human mucosa. The female and mucosa fold-like structure elements were also reflected to the VEGF-A-positive cells density of the epithelial layer in our results. At least, there are the correlation between Ki-67-positive cell density and VEGF-A-positive cells density in human mucosa. In our PCA analysis, the positive contributions for denture conditions, such as tooth marks (positive correlation; ME, $\mathrm{MCo}$, gender) is the most important elements, while negative contributions include cell differentiation (Ki-67) (D2-40, denture, VEGF-C, VEGF-E, ME). In component 1, positive contributions were defined the VEGF-C, gender, and the ME, in contrast, negative contributions from cell differentiation in component 1 . Moreover, component 2 was defined by the expression of CD31and tooth marks, which had positive contributions in our results. According to the PCA maps, dentures and D2-40 expression were also correlated. The mucosa structure, VEGF and gender elements were also same correlative groups in the our PCA map results. Therefore, the tooth mark (denture) affects to the angiogenesis (CD31, D2-40) and the ME in association with aging. In contrast, cell differentiation marker Ki-67 affect to the MCo in association with the gender and VEGF-A. Stimulation by tooth marks eventually may break these relationships of angiogenesis, cell differentiation and aging and, as a result, triggers carcinoma in the oral cavity.

\section{References}

1. Raica M, Cimpean AM, Ribatti D. Angiogenesis in premalignant conditions. Eur J Cancer 2009;45:1924-34.

2. Siemerink MJ, Klaassen I, Vogels IM, Griffioen AW, Van Noorden CJ, Schlingemann RO. CD34 marks angiogenic tip cells in human vascular endothelial cell cultures. Angiogenesis 2012;15:151-63.

3. Tung JJ, Tattersall IW, Kitajewski J. Tips, stalks, tubes: notchmediated cell fate determination and mechanisms of tubulogenesis during angiogenesis. Cold Spring Harb Perspect Med 2012;2:a006601.

4. Carlile J, Harada K, Baillie R, Macluskey M, Chisholm DM, Ogden GR, et al. Vascular endothelial growth factor (VEGF) expression in oral tissues: possible relevance to angiogenesis, tumour progression and field cancerization. J Oral Pathol Med 2001;30:449-57.

5. Pazouki S, Chisholm DM, Adi MM, Carmichael G, Farquharson M, Ogden GR, et al. The association between tumour progression and vascularity in the oral mucosa. J Pathol 1997:183:39-43.

6. Bazarsad S, Zhang X, Kim KY, Illeperuma R, Jayasinghe RD, Tilakaratne WM, et al. Identification of a combined biomarker for malignant transformation in oral submucous fibrosis. J Oral Pathol Med 2017;46:431-8.

7. Pyziak L, Stasikowska-Kanicka O, Danilewicz M, WągrowskaDanilewicz M. Immunohistochemical analysis of mast cell infiltrates and microvessel density in oral squamous cell carcinoma. Pol J Pathol 2013;64:276-80.

8. Ogawa T, Ogimoto T, Sumiyoshi K, Koyano K. Pressure-pain threshold of oral mucosa and its region-specific modulation by pre-loading. J Oral Rehabil 2003;30:1062-9.

9. Isobe A, Sato Y, Kitagawa N, Shimodaira O, Hara S, Takeuchi S. Influence of denture supporting tissue properties on pressure-pain threshold--measurement in dentate subjects. J Prosthodont Res 2013;57:275-83.

10. Cassar-Malek I, Picard B, Kahi S, Hocquette JF. Relationships between thyroid status oxidative metabolism and muscle differentiation in bovine fetuses. Domest Anim Endocrinol 2007;33:91-106.

11. Krajewska M, Smith LH, Rong J, Huang X, Hyer ML, Zeps N, et al. Image analysis algorithms for immunohistochemical assessment of cell death events and fibrosis in tissue sections. J Histochem Cytochem 2009;57:649-63.

12. Stănescu R, Didilescu AC, Jianu AM, Rusu MC. Angiogenesis in the reparatory mucosa of the mandibular edentulous ridge is driven by endothelial tip cells. Rom J Morphol Embryol 2012;53:375-8.

13. Tsuruoka M, Ishizaki K, Sakurai K, Matsuzaka K, Inoue T. Morphological and molecular changes in denture-supporting tissues under persistent mechanical stress in rats. J Oral Rehabil 2008:35:889-97.

Received for publication: 2 December 2019. Accepted for publication: 17 February 2020.

This work is licensed under a Creative Commons Attribution-NonCommercial 4.0 International License (CC BY-NC 4.0).

(C) Copyright: the Author(s), 2020

Licensee PAGEPress, Italy

European Journal of Histochemistry 2020; 64:3095

doi:10.4081/ejh.2020.3095 